\title{
Nanocrystalline Diamond Films Grown by Microwave Plasma Chemical Vapor Deposition and Its Biocompatible Property
}

\author{
Jihan Yang, Yongping Zhang \\ Faculty of Materials and Energy, Southwest University, Chongqing, China \\ Email: zhangyyping@yahoo.com
}

How to cite this paper: Yang, J.H. and Zhang, Y.P. (2018) Nanocrystalline Diamond Films Grown by Microwave Plasma Chemical Vapor Deposition and Its Biocompatible Property. Advances in Materials Physics and Chemistry, 8, 157-176. https://doi.org/10.4236/ampc.2018.84011

Received: September 23, 2017

Accepted: April 6, 2018

Published: April 9, 2018

Copyright ( $\odot 2018$ by authors and Scientific Research Publishing Inc. This work is licensed under the Creative Commons Attribution International License (CC BY 4.0).

http://creativecommons.org/licenses/by/4.0/

\begin{abstract}
Due to its unique properties such as high hardness, light transmittance, thermal conductance, chemical stability and corrosion resistance, diamond has drawn tremendous attention in last two decades. These specific properties made diamond film a promising material for cutting tools, microwave windows, heat sinks for electronic devices and diamond electrodes. However, the diamond film with grain sizes at microscale usually exhibits high surface roughness and hinders its applications in the microelectro mechanical system (MEMS) and biological field because it is difficult to be polished by mechanical and chemical methods. With the development of the chemical vapor deposition, the nanocrystalline diamond (NCD) film has been fabricated and found new applications. The grain size of NCD film is in the range of 10 to $100 \mathrm{~nm}$, which inherits the properties of the diamond and possesses the unique properties of the nanoscale materials, and the morphology of the NCD film is granular or needle-like structure. The microwave plasma chemical vapor deposition (MPCVD) has been regarded as the most promising method to deposit NCD film at low temperature. Compared to the hot filament CVD, MPCVD can grow high quality NCD film avoiding of the contamination from the filament materials. The MPCVD technique has high plasma density to activate carbonaceous compound and grow NCD film in high growth rate and low substrate temperature. The unique properties of NCD film, such as the superior electrical, mechanical and biological properties facilitate their application in various fields. The biological application, especially as a biocompatible coating, mainly includes the joint replacement implants and protective coatings and the ophthalmological prosthesis.
\end{abstract}

\section{Keywords}

Nanocrystalline Diamond Films, Microwave Plasma Chemical Vapor 
Deposition, Biocompatible Property

\section{Introduction}

Diamond as an outstanding material for its unique properties, such as high hardness, chemical inertness, high field emission, high carrier mobility, has attracted much attention over the decades. But natural diamond is difficult to be engineered into many physical configurations required for the desired properties. With the development of the chemical vapor deposition (CVD), which can synthesize the nanocrystalline diamond (NCD) with the precursors of hydrocarbon and hydrogen. NCD inherited the properties of the bulk diamond, and also had nano-materials' properties. NCD film has great potential of applications such as surface acoustic wave, microelectro mechanical systems (MEMS) and biomedical implants.

After William G. E. synthesized diamond by chemical vapor deposition in 1962, researchers and scientists made effort to improving this method [1]. Various CVD methods had been developed, such as low pressure chemical vapor deposition, hot flame chemical vapor deposition, radiofrequency plasma chemical vapor deposition and microwave plasma chemical vapor deposition. Among all these methods, the microwave plasma chemical vapor deposition (MPCVD) was widely used in laboratory and industry. The microwave produced high density and high temperature plasma with electron density in the order of $10^{11} \mathrm{~cm}^{-3}$, which is beneficial to deposit high quality diamond. For microwave plasma deposition technology, electron cyclotron resonance plasma could deposit diamond films at pressure of some hundredths to thousandths torr. Zarrabian et al. concluded that they observed NCD film with pressure of 2.7 mTorr [2]. Kouzuma et al. also reported diamond film could be deposited at pressure of 1 mTorr [3].

The research and development of NCD films have been developing rapidly in the last decades and new applications emerged continuously. This paper is an attempt to review the structural characteristics, properties, growth techniques of MPCVD and biocompatible applications. Section 2 describes the structural characteristics of NCD compared with microscale diamond films. Section 3 deals with the excellent biocompatible properties. Section 4 discusses the advantages of MPCVD growth techniques. Section 5 introduces the compatible applications of NCD films. In the final section, summary and research prospect are provided.

\section{Structural Characteristics of Nanocrystalline Diamond}

Because the carbon atoms are linked by the strong covalent bond, the diamond films possess a series of outstanding properties, for instance high hardness, high thermal conductivity, excellent electrical performance and chemical inertness. 
Figure 1(a) showed the crystal structure of the diamond, composing of $\mathrm{sp}^{3}$ hybridized $\mathrm{C}$ atoms tetrahedrally bonded to form a lattice in which two sets of face centered cubic along the body diagonal translate a quarter of the distance of lattice constant. These superior properties made diamond film applied broadly in the wear resistant device, MEMS, flat panel display and medicine. However, the large grain size and the coarse surface of the microcrystalline diamond film make great trouble in polishing and hindering the application. NCD film has dual properties of diamond and nanomaterials, and has more advantages than ordinary diamond film, such as low friction coefficient, low surface roughness and strong field emission properties. Moreover, the poor electronic mobility of intrinsic NCD film can be tuned by element doping. The $\mathrm{n}$ - and p-type semiconductor NCD film can be obtained by doping nitrogen and boron in the diamond lattice, respectively. The doped NCD film has outstanding field electron emission properties, low onset field and high current density which make NCD film has unparalleled advantages in field emission electron (FEE) application than other materials. Meanwhile, the resistivity of the doped film is a function of the concentration of the gas precursor.

The crystalline grains of NCD film, consisting of diamond phase more than $95 \%$, are between tens to hundreds nanometres in size. Many studies showed that the NCD film had characteristics of high hardness, low friction coefficient, and low surface roughness. The hardness of the NCD film was slightly lower than that of the microcrystalline diamond film, while the friction coefficient and surface roughness of the NCD film were lower one order of magnitude than that of the microcrystalline diamond film, which made it become an excellent mechanical coating material. Especially, the NCD film has favorable biocompatible and bioinert property which have been considered as the biomaterial of the $21^{\text {st }}$ century. The bio-properties of the NCD film had been investigated extensively. The performances of the cells' adhesion, cultivation, proliferation on the NCD film were influenced by the surface modification of the film.
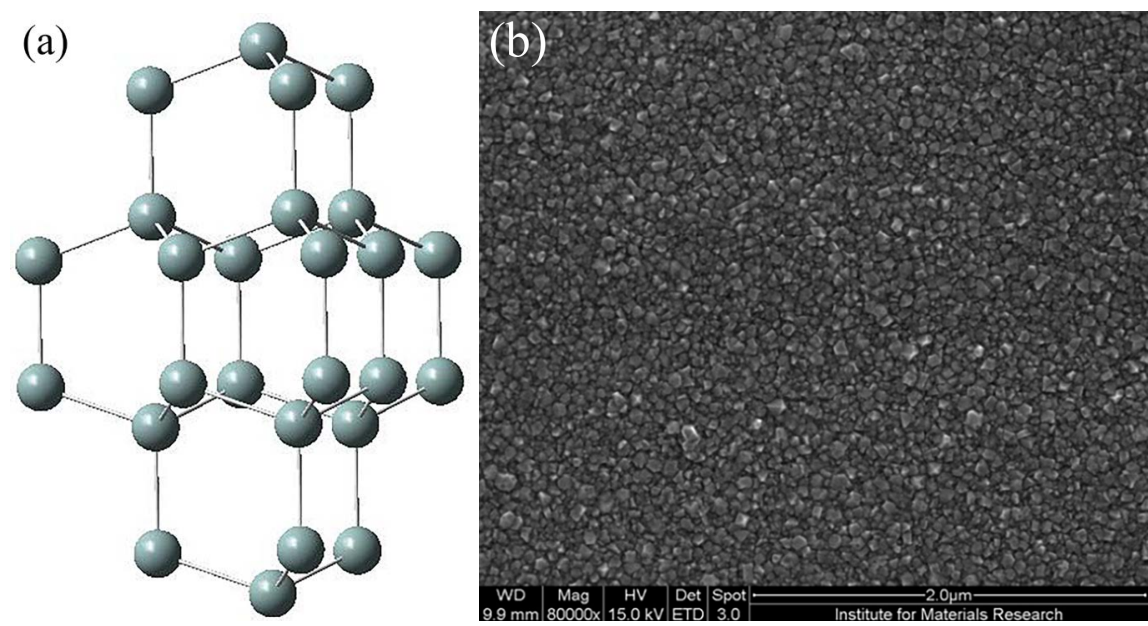

Figure 1. The crystal structure of the diamond (a) and scanning electron image of nanocrystalline diamond film (b). 
The surface topography, surface functionalization and the doping level affected greatly the cell growth. According to the reports, the adsorption of the protein was high on rough NCD film comparing that of the low roughness NCD film. The proliferation of the neuroblastoma on the hydrogen terminated NCD film and the differentiation of the neural stem cell on the boron doped NCD films also had been demonstrated. Therefore, the NCD film can be used in the biological application on account of the excellent biocompatible properties of the film. The joint replacement implants and protective coatings, such as the protective coating of the hip prosthesis and the artificial heart valve, had been widely investigated. While the application in the ophthalmology was rarely reported. Recently, the NCD film found potential application inn ophthalmological prosthesis. The NCD film could help to restore the sight and deposit on the retinal implant as long-term coating. NCD film was smooth surface and with non-diamond phase deposited by MPCVD in carbon-lean and hydrogen-rich environment. Figure 1(b) presented the scanning electron micrograph of the surface [4]. The NCD film had the clear facet crystal grain, and the surface roughness was low. Grain size in the film was between 50 and $100 \mathrm{~nm}$.

Raman spectrum is sensitive to carbon nanophase, thus becomes the best tool for analyzing the chemical component of carbon materials. Figure 2 showed the typical Raman spectra of NCD films [5]. These films were deposited by the microwave plasma CVD system using the $\mathrm{CH}_{4} / \mathrm{H}_{2} /$ Air as the gas precursor, and the inset presented the thickness of the films corresponding to the deposition time. Six peaks were observed in the Raman spectrum. There are trans-polyacetylene (t-PA) peaks, which refered to the grain boundary phase in the nanodiamond [6]. The $\mathrm{D}$ band was attributed to the disorder carbon bond and $\mathrm{G}$ band related to the graphite phase and amorphous carbon with Raman shift at $1360 \mathrm{~cm}^{-1}$ and $1580 \mathrm{~cm}^{-1}$, respectively. The fingerprint of the diamond phase was at $1332 \mathrm{~cm}^{-1}$ [7]. The intensity of the t-PA and G-band peak increased with the thickness indicating the chemical structures of grain boundary were changed. For instance, the similar Raman spectrum was found in the film grown in the methane-lean.

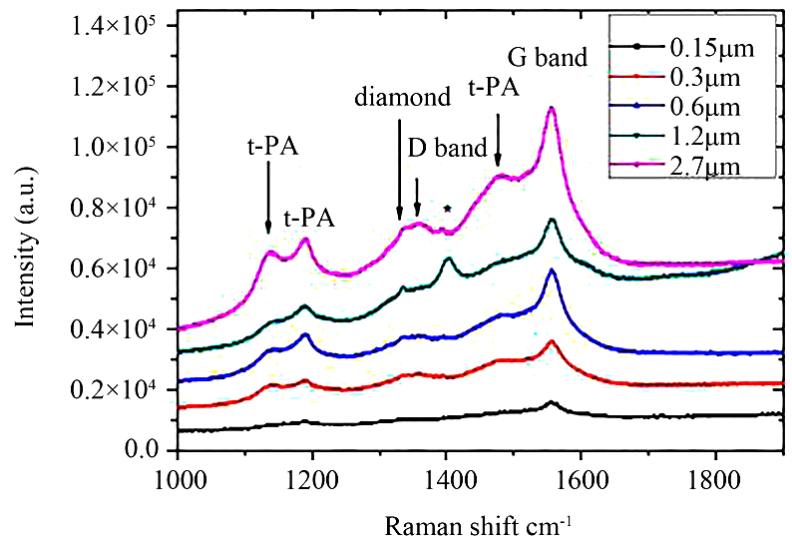

Figure 2. Raman spectra of the NCD films of 0.15 to $2.7 \mu \mathrm{m}$ thickness. 
Revati et al. deposited the films at the condition of the $\mathrm{CH}_{4}(1 \%) / \mathrm{Ar}$ with microwave power of $1200 \mathrm{~W}$, and nitrogen-rich environment. Sankaran et al. used $\mathrm{N}_{2}(94 \%) / \mathrm{CH}_{4}(6 \%)$ as the gas precursor with the microwave power at $1200 \mathrm{~W}$ [8]. The similarity of the Raman spectra showed the similarity of the chemical structure.

Another category of NCD is the ultra-nanocrystalline diamond (UNCD) [9] [10] [11]. To distinguish the difference between NCD and UNCD, UNCD is defined as diamond films with the grain size in the range of 2 to $10 \mathrm{~nm}$ and the film's boundary containing the $\mathrm{sp}^{2}-\mathrm{C}$. The surface of UNCD is very smooth, moreover, the surface roughness do not increase with the thickness of the film [12] [13]. The typical UNCD film is grown in the argon rich and hydrogen poor environment, with a typical ratio of argon, hydrogen and methane is $99 \%-95 \%$, $1 \%-4 \%, 1 \%-2 \%$, respectively. Therefore, many research teams were attracted by the potential applications of NCD and UNCD and started to study the synthesis, characterization and application of NCD and UNCD.

\section{Properties of Nanocrystalline Diamond}

The hardness of NCD film synthesized by CVD is approximately close to that of the natural diamond, while the friction coefficient is lower than that of the natural diamond. Therefore, NCD film is an excellent coating material, such as cutting tool and dry friction material. Liu et al. prepared the UNCD films on the Si and $\mathrm{Si}_{3} \mathrm{~N}_{4}$ substrates with different pre-treatments of the substrate surface [14]. They found the hardness of the films grown on the different substrates was similar. Many researches had verified that NCD films had characteristic of high hardness, low friction coefficient, and low surface roughness. Fendrych et al. reported the NCD films deposited by the plasma enhanced linear antenna microwave CVD system via the gas mixture of $\mathrm{H}_{2}-\mathrm{CH}_{4}-\mathrm{CO}_{2}$ [15]. The roughness is below $30 \mathrm{~nm}$ and the friction coefficient of the NCD film below 0.8. Bogdanowicz et al. investigated the surface roughness of the film affected by the thickness of the $\mathrm{TiO}_{2}$ interlayer deposited via magnetron sputtering [16]. They found the roughness of the film decreased with increasing of the thickness of the interlayer, and the roughness of the film was $18 \mathrm{~nm}$ after depositing the interlayer for $1 \mathrm{~h}$. Li et al. studied the different metallic seed layers affected on the properties of NCD film [17].

The Mo, Ti, W layers were deposited on the Si wafer and grown the naocrystalline diamond film by microwave plasma CVD method. The surface root mean square roughness of the NCD film on the Mo, Ti, W layer was 20.8, 23.7, 19.4 $\mathrm{nm}$, respectively. These unique properties of NCD film, such as friction and wear properties, have more prominent than microcrystalline diamond film. Table 1 presented the comparison of some properties of NCD and the microcrystalline diamond. The surface of NCD is smoother and the friction coefficient is lower than these of the mircocrystalline diamond. Meanwhile, NCD film also has good wear resistance and high mechanical strength, which is an ideal material for components in MEMS, nanoelectro mechanical system (NEMS) [19]. 
Table 1. Comparison of some properties of the nanocrystalline diamond and the microcrystalline diamond.

\begin{tabular}{ccc}
\hline & Nanocrystalline diamond & Microcrystalline diamond \\
\hline Electronic & Poor Mobility $[4]$ & Semiconductor properties \\
Hardness [GPa] & $35-75[18]$ & 100 \\
Friction coefficient & $0.02[7]$ & 0.1 \\
Young's modulus [GPa] & $1100[18]$ & 1120 \\
Grain size [nm] & $10-100$ & $>10,000$ \\
\hline
\end{tabular}

Diamond's band gap is the widest in all semiconductor materials, and the band gap of high purity diamond is $5.5 \mathrm{eV}$. With the increases of impurity concentration, the band gap of diamond decreases. When the III group (B, Ga, As) and $\mathrm{V}$ group elements $(\mathrm{N}, \mathrm{P})$ are doped into the NCD film, the resistivity of NCD film decreases. These excellent semiconductor properties make it possible for doped NCD film applied in the field of semiconductor device. The doping method of NCD film mainly includes film growth process doping (CVD doping) and ion implantation. The CVD doping is the incorporation of doped atoms into the film during the CVD growth of diamond film, which is the most commonly used doping method for diamond doping. While, ion implantation doping can effectively control the impurity type and concentration in the diamond film, which widely used in the semiconductor process. Meanwhile, ion implantation doping can also control the density and depth distribution of impurities in diamond film. However, it is important to control the appropriate ion implantation process and the subsequent annealing process, since ion implantation process will inevitably cause defects damaging the lattice of diamond. Generally, the electrical properties of the NCD and UNCD film can be improve by doping to obtain p- and n-type semiconductor film [20] [21]. For p-type NCD films, boron is the only element can be easily introduced into the lattice of diamond films without apparently changing the structure. Boron has small atomic radius, which is shallow acceptor impurity of diamond, so the resistivity of diamond film after boron doping has declined sharply, leading to the improvement of the conductive performance of the film [22] [23]. The suitable concentration of boron doping not only improves the conductivity of diamond film, but also improves the crystal quality and morphology by adding a small amount of boron to diamond film. Some other elements, like S and As, are unsuccessfully doped in NCD film due to its geometric and energetic properties are not similar.

The field emission property of boron-doped NCD film had been presented in Figure 3(a), in which the onset field was demonstrated to be $0.36 \mathrm{~V} / \mu \mathrm{m}$ [24], and the doped diamond film had better field emission properties. At present, the study on the boron doped p-type NCD film was relatively mature, whose resistivity reaching $10^{-2} \Omega \cdot \mathrm{cm}$ could meet the device requirement. In general, n-type NCD film is doped by nitrogen which can be deposited by the microwave plasma CVD with the gas precursor $\mathrm{CH}_{4} / \mathrm{N}_{2}, \mathrm{CH}_{4} / \mathrm{H}_{2} / \mathrm{N}_{2}, \mathrm{Ar} / \mathrm{CH}_{4} / \mathrm{N}_{2}, \mathrm{Ar} / \mathrm{CH}_{4} / \mathrm{N}_{2} / \mathrm{H}_{2}$. 


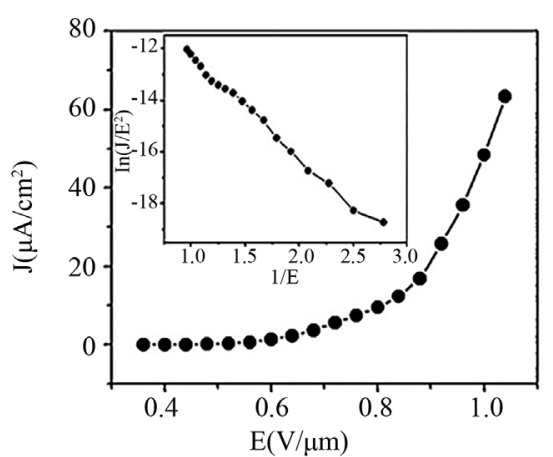

(a)

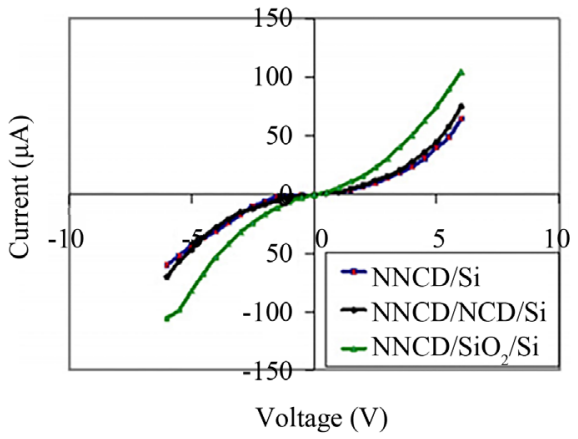

(b)

Figure 3. (a) The field emission current density versus the applied field plot of the boron-doped nanodiamond film; (b) I-V curves of the different structures of the n-doped nanocrystalline diamond (NNCD) film.

Nitrogen doping is one of the important methods to improve the electrical properties of diamond film. Due to the doping of the nitrogen impurities, the chemical states of the diamond grain boundary and the band structure had been changed to improve the conductivity of the diamond film. Furthermore, the nitrogen doped diamond film has excellent chemical stability and field emission characteristics. Various results had reported the improvement of the electrical properties of the n-type nanocrystallline diamond film. For instance, Ikeda et al. had grown NCD film on the polished silica with a mixture of $69 \% \mathrm{Ar}-30 \% \mathrm{~N}_{2}-1 \% \mathrm{CH}_{4}$ [21]. They investigated the resistivity of the film at different deposition temperature of $1100 \mathrm{~K}$ and $1200 \mathrm{~K}$, as a function of the $\mathrm{N}_{2}$ concentration. For both the deposition temperature, the resistivity of the film decreased with the increase of the $\mathrm{N}_{2}$ concentration and saturated for $\mathrm{N}_{2}$ concentration more than $30 \%$. With the increase of nitrogen content in the reaction gas source, the grain boundary width of the diamond increased, and the increase of the grain boundary is the main reason for the change of the conductivity of the film. Hu et al. reported three different layer structures of the nitrogen-doped NCD film grown on the $\mathrm{Si}$ wafer by microwave plasma CVD method [25]. Nitrogen doped NCD layer was deposited by the recipe $\mathrm{Ar} / \mathrm{N}_{2} / \mathrm{CH}_{4} / \mathrm{H}_{2}$ with a ratio of $83 \% / 15 \% / 1 \% / 1 \%$. Figure 3 (b) showed the I-V curves of the n-doped NCD films with different structures. Apparently, the $\mathrm{NNCD} / \mathrm{SiO}_{2} / \mathrm{Si}$ had the highest I/V ratio. Recently, Yuan et al. used triethylamine as a new nitrogen source which was liquid dissolved in the methanol to synthesize nitrogen doped UNCD film on Si substrate by microwave plasma CVD [26]. They made the triethylamine and methanol vapor and sent the gas into the growth chamber with the Ar. Three different UNCD films were synthesized with different substrate temperatures and the electrical conductivity as well as the electron field emission were investigated. The conductivity of the film increased with the increase of the substrate temperature, and the electrical conductivity of the film deposited at $890^{\circ} \mathrm{C}$ was higher than the traditional nitrogen-doped UNCD film though $\mathrm{N}_{2}$ addition. The best electron field emission (EFE) property of the film was obtained with the substrate temperature 
at $830^{\circ} \mathrm{C}$, having the lowest turn on field of $3.4 \mathrm{~V} \cdot \mu \mathrm{m}^{-1}$ and the highest current density of $8.0 \mathrm{~mA} \cdot \mu \mathrm{m}^{-2}$. In summary, the electrical properties of NCD film could be effectively tuned by the doping, which made the film a great potential material for the applicant in the MEMS and NEMS.

So far, a large number of studies had showed that the diamond film deposited by the chemical vapor deposition had similar biocompatibility with commonly implants [27] [28] [29] [30]. Owing to the C atom can connect to H, O, hydroxyl and carbocxyl and other molecules by chemical bond to form stable terminated surface, which can graft NCD film with small molecular functional groups to realize biological function of NCD film's surface [31] [32]. NCD film has favorable biocompatible and bioinert which has been considered as the biomaterial of the 21st century. Many publications had described the bio-properties of NCD film and found the morphology, the surface termination and element doping of the NCD film affected the cell adhesion, cultivation, proliferation. Recently, Verdanova et al. reported the NCD film promoted the cell proliferation [33]. They found that carbon materials had enhanced the cultivation of the cell than the polystyrene. What's more, the better cell adhesion was found on NCD compared to the graphene layer. But the better cell proliferation was found on the graphene layer. Alcaide et al. investigated the surface roughness and the doping level of NCD film affected the protein adsorption and the adhesion of the fibroblasts [34]. The surface roughness of NCD film deposited on the glass substrate was about $28 \mathrm{~nm}$, and the surface roughness of NCD film deposited on the Ti substrate was about $110 \mathrm{~nm}$. The protein adsorption on the high roughness NCD film was higher than that of the low roughness NCD film in their experiments. Furthermore they used the boron as the doping element, they found that the protein adsorption of the boron doped NCD film was higher than that of the NCD film in both high surface roughness and low surface roughness. Figure 4 showed the SEM images of the cells cultured on the film after $24 \mathrm{~h}$. In general, the adhesion of the cell on the high roughness of the film was better than that on the film with the low roughness, whether the untreated and doping or not. Among these films, the untreated film presented the highest level of protein adsorption. Furthermore, there appears no difference between the undoped and doped films. While the hydrogen termination surface of NCD film had hydrophobic and conductive properties and the oxygen termination surface of the NCD film had hydrophilic and electrical resistive properties, such unique properties made NCD films use as cell-based biosensors [35] [36].

Cellular adhesion is very important for many biological processes, because the adhered cells can sense, integrate, interpret and respond to some extracellular signals due to specific interaction between features preset on the NCD-based surface and cell surface receptors [37]. Bajaj et al. demonstrated that the rat pheochromocytoma cells were cultivated on different substrates, such as UNCD film, silicon and platinum and investigated the cell adhesion, proliferation and growth of the cell on those substrates [38]. As a result, the cell on the UNCD 

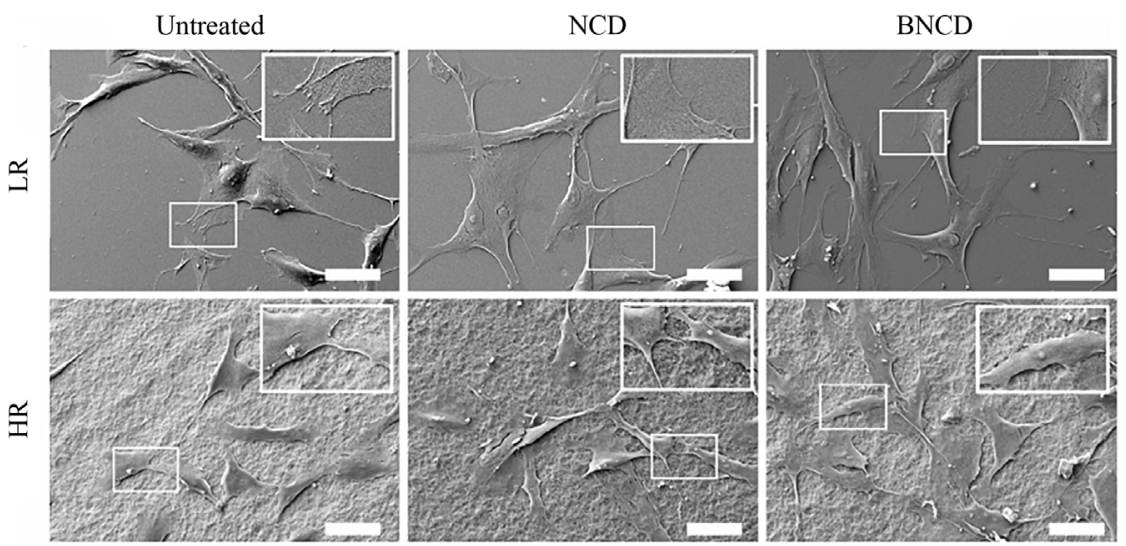

Figure 4. The SEM images of the cell culture on the different films after $24 \mathrm{~h}$. The inset of the images is the high-resolution images of the zone marked by rectangle and the scale bar indicated as $50 \mu \mathrm{m}$.

film possessed the maximum cell number and spreading. According to published reports, the various cells could be cultivated on the NCD and UNCD film [39] [40] [41]. Chen et al. showed the differentiation of the neural stem cells could be effected by the surface properties of the UNCD film [42]. The UNCD film with very small grain and low surface roughness coating on the implantable devices for neural application. Tong et al. investigated the nitrogen doped UMCD film as the high capacitance neural interfaces [43]. The rat cortical neurons were cultivated on the N-UNCD, which was treated by the oxygen plasma, and the growth of the neurons was affected by the surface roughness. Therefore, the neuron interface fabricated by the oxygen plasma treated N-UNCD with appropriate surface roughness was high performance and long-term stability. Vaitkuviene et al. investigated the different surface terminations affected and maintained the growth of the human neuroblastoma cells [44]. The NCD film was deposited on the fused silica by the microwave plasma chemical vapor deposition, and used the UV induced ozone treatment to obtain the oxygen terminated NCD film. As a result, the adhesion of the cells on all NCD film increased as the standard tissue cultured polystyrene, which indicated the cells adhered both on the hydrogen terminated and oxygen terminated surface as well. But other groups found that cells cultured on different terminated surface presented various appearances. Lechleitner et al. reported the human renal epithelial cell was suppressed by the hydrogen terminated NCD surface. On the oxygen terminated NCD surface, however, the adhesion of the cell was improved [45]. The UNCD film also used as the substrate for the growth and proliferation of the cells, the suitability and noncytotoxicity of these films had been investigated [46]. Auciello et al. demonstrated that the surface of UNCD could be used to grow and differentiate the embryonic stem cells, and then the embryonic stem cells differentiated into retina photoreceptors to treat the blind patients caused by the death of the photoreceptors [47]. Eventually, NCD film had gradually applied in the media field and became excellent media material. In the field of 
medicine, NCD film had attracted extensive attention due to its good biocompatibility, corrosion resistance, high surface area and ease to modify.

In summary, the semiconducting NCD film was gained by doped the elements, such as nitrogen, boron. The n- and p-type NCD film both showed the excellent electrical properties. The mechanical properties were affected by different metal layer also had been shown, NCD film had great hardness and low surface roughness. The biological properties had been discussed, such as the adhesion, cultivation, proliferation of the cell on the NCD film.

\section{Microwave Plasma Chemical Vapor Deposition}

The chemical vapor deposition process is mainly involved gas phase react on the solid surface and film growth. Chemical vapor deposition is simple to operate and can deposit high quality NCD film, which greatly facilitate the development and application of NCD film. There are many deposition methods to deposit NCD film, for instance microwave plasma, hot filament, combustion flame, direct current arc CVD. Among those methods, hot filament chemical vapor deposition and MPCVD are commonly used to deposit the nanocrtystalline diamond film. Hot filament CVD commonly used to deposit large area of substrate with up to $300 \mu \mathrm{m}$ in the diameter. However, the sample deposited by the hot filament CVD may be contaminated by the filament material, and the growth rate of the hot filament CVD is fairly low. MPCVD used high density plasma to decompose carbonaceous compound, such as $\mathrm{CH}_{4}, \mathrm{C}_{2} \mathrm{H}_{2}, \mathrm{CO}_{2}$, and hydrogen into $\mathrm{C}^{4-}$ species, then hydrogen atom and activated carbon react on the substrate surface. MPCVD is a widely used technique for depositing NCD film at present.

Substrate temperature is a vital factor for diamond deposition. NCD film was deposited by MPCVD at substrate temperature between $300^{\circ} \mathrm{C}$ to $1000^{\circ} \mathrm{C}$. Subranmanian et al. reported the substrate temperature was maintained at $800^{\circ} \mathrm{C}$ during the deposition process [48]. The deposition parameters are $\mathrm{CH}_{4} / \mathrm{H}_{2} / \mathrm{N}_{2}$ as the reactive gas, the microwave power maintained at $550 \mathrm{~W}$ and the pressure maintained at 28 Torr. Das et al. kept the substrate temperature at $200^{\circ} \mathrm{C}$ to $300^{\circ} \mathrm{C}$ without external heating [49]. The substrate was automatically heated due to the substrate close to the plasma. A stainless steel multi-mask system was used to protect the substrate from the damage of the active plasma. The XPS experiment found the film contained $84.77 \% \mathrm{sp}^{3}$ phase, indicating the good quality of the film. Sankaran et al. reported the nitrogen incorporated ultraNCD film with the gas precursor $\mathrm{N}_{2}(96 \%)$ and $\mathrm{CH}_{4}(4 \%)$ by microwave plasma CVD method [50]. A negative bias with $250 \mathrm{~V}$ was applied to the substrate in the growth process, and the substrate temperature was around $450^{\circ} \mathrm{C}$ heated by the plasma. The microstructure of the film is a function of the nitrogen incorporated UNCD film. Lin et al. studied the incorporation of $\mathrm{H}_{2}$ into Ar plasma and investigated the vary of the microstructure of NCD film with a gas mixture of the $\mathrm{CH}_{4} / \mathrm{Ar} / \mathrm{H}_{2}$ and the flow rate of the $\mathrm{CH}_{4}$ maintained at $1 \mathrm{sccm}$ (standard state cubic per 
minute), and the $\left(\mathrm{Ar}-\mathrm{x} \% \mathrm{H}_{2}\right)=99 \%$. The substrate temperature was kept at between $460^{\circ} \mathrm{C}$ and $595^{\circ} \mathrm{C}$ without external heater [51]. It is generally believed that the low substrate temperature is favorable for improving the re-nucleation rate during deposition, which refines the grain size to achieve high flatness, high quality and large area of nanocrysatlline diamond film. However, the high substrate temperature is benefited to the growth rate. Thus, the substrate temperature was controlled in a certain range in the process of deposition of NCD films to obtain high quality NCD film.

Another advantage of MPCVD is that the NCD film has relatively high growth rate, due to high density plasma and plasma composition. Tang et al. presented that the NCD film was synthesized using $\mathrm{CH}_{4} / \mathrm{H}_{2} / \mathrm{O}_{2} / \mathrm{N}_{2}$ as the gas precursor with high growth rate of $2.6 \mu \mathrm{m} / \mathrm{h}$ [52]. The process used high microwave power of $3 \mathrm{~kW}$ and the substrate temperature at $700^{\circ} \mathrm{C}$. Tang et al. reported that NCD film was deposited with high microwave power and high growth rates [53]. The typical parameters used to deposit microcrystalline diamond film are with the microwave power of $3 \mathrm{~kW}$ and the ratio of $\mathrm{CH}_{4} / \mathrm{H}_{2}$ is $4 \%$. The average grain size of NCD film deposited by their experiment is $47 \mathrm{~nm}$. When the $\mathrm{N}_{2}$ was introduced into the gas precursor, the growth rate of the film increased to $5.4 \mathrm{~nm} / \mathrm{h}$. While the parameter of the experiment changed to the microwave power of $4.0 \mathrm{~kW}$ and the flow rate of the $\mathrm{N}_{2}$ maintained at $1.6 \mathrm{sccm}$, the growth rate varied from $5.4 \mathrm{~nm} / \mathrm{h}$ to $9.6 \mathrm{~nm} / \mathrm{h}$, with the average grain size of $34 \mathrm{~nm}$. Recently, a novel method, in-liquid plasma chemical vapor deposition, deposited NCD film with a remarkable growth rate [54]. Harada et al. reported the growth rate of the NCD film deposited by the in-liquid plasma chemical vapor deposition was calculated to be $8.5 \mu \mathrm{m} / \mathrm{h}$. Table 2 showed the different growth rates of the NCD film by different groups. It is clear that MPCVD achieved high growth rate.

MPCVD generated high density plasma, which can enhance the concentration of the active species and be benefit for depositing film. And this method can generate plasma without the electrode, thus deposit high quality and high purity NCD film by inhibiting impurities introduce.

In summary, the general method of the NCD film was the chemical vapor deposition (CVD) including the hot filament chemical vapor deposition and the microwave plasma chemical vapor deposition. The HFCVD could deposit the

Table 2. Comparison of the growth rate of the NCD film reported in the literature and produced by different groups.

\begin{tabular}{ccccc}
\hline $\begin{array}{c}\text { Grain size } \\
(\mathrm{nm})\end{array}$ & $\begin{array}{c}\text { Growth rate } \\
(\mu \mathrm{m} / \mathrm{h})\end{array}$ & $\begin{array}{c}\text { Reactant } \\
\text { gases }\end{array}$ & $\begin{array}{c}\text { Growth } \\
\text { method }\end{array}$ & Reference \\
\hline 60 & 2.6 & $\mathrm{CH}_{4} / \mathrm{H}_{2} / \mathrm{N}_{2} / \mathrm{O}_{2}$ & MPCVD & {$[52]$} \\
52 & 0.3 & $\mathrm{CH}_{4} / \mathrm{H}_{2}$ & MPCVD & {$[55]$} \\
82 & 1.16 & $\mathrm{CH}_{4} / \mathrm{H}_{2} / \mathrm{Ar} / \mathrm{CO}_{2}$ & MPCVD & {$[56]$} \\
\hline
\end{tabular}


large area film, but the HFCVD was severe to the substrate and the impurity would introduce to the film. The MPCVD was widely used in the laboratory and factory since MPCVD had the advantages of the low temperature, high growth rate and low deposition pressure.

\section{Biocompatible Application}

Nanocrystalline diamond film has attract the attention of many researchers in materials, physics, biology, electronic and other fields for the outstanding physical and chemical properties, excellent biocompatibility, high elastic modulus and unique electric properties. The unique properties make NCD film have potential application in the field of physics science, biomedicine and its related fields. The application of NCD film had been one of the hot research topics in materials, physics and biology in the $21^{\text {st }}$ century [57] [58] [59] [60]. In general, the common diamond film was used as the protective coating for the cutting tool and the wear resisting coating for the bearings. Recently, UNCD was used as the protective coating for the AFM cantilever [61]. Moreover, the NCD had been used as the optical material [62] [63] [64]. However, NCD film and UNCD film have good chemical inertness, non-toxic, excellent wear resistance and biological, the films can be hydrophilic and hydrophobic by changing the surface dangling bonds. Combined with their high surface smoothness, the film can be used as an encapsulation layer for artificial joints, artificial hearts, artificial teeth and other artificial medical devices. Thus, the harmful effect of the implanted equipment for the human body can be effectively overcome by isolating the inorganic layer and the biological organic layer. The biocompatibility and bioinertness of the NCD and UNCD films make these films as the ideal candidates for the medical application.

Each year many people suffered arthritic diseases and trauma induced by accidents and accepted the hip and knee joint replacement. The challenging of the replacement is that the surface of the implant may produce the wear debris particles which can cause the osteolysis and aseptic loosening. While the implant coated by the NCD is benefit to reduce the wear of the implant and avoid the adverse reaction with the surrounding tissues. Actually, the diamond coating had been used for various medical devices for the purpose of protecting the device and ensured the lifetime of the devices, such as the joint prostheses. Due to the properties of the biocompatibility, bioinert, high hardness and low friction, alternative application the NCD film was the joint prostheses. Amaral et al. deposited the NCD film on the $\mathrm{Si}_{3} \mathrm{~N}_{4}$ substrate and the pin-on-plate experiment was carried out. The NCD film was the good candidate for the protective coating of the hip prosthesis, femoral head [65]. The NCD film could avoid the attachment of the cells, thus the NCD film could be deposited on the artificial heart valve for the protecting coating. NCD film deposited the heart valve ring as the protecting coating [66], with the dense NCD film covered on the Ti surface. The Raman spectra showed the film still covered well on the heart valve ring after the 
mechanical fatigue test.

Another potential application of the UNCD films is the ophthalmological prosthesis. The UNCD films were coated on an implantable microchip that inside human eyes for restoring the sight. The protective coatings of the implantable microchip protected the microchip from the eye fluid and protect eye from the hip material, therefore the UNCD film's own the bioinertness and biocompatibility were the reliable candidate for the ophthalmological prosthesis. Common artificial retinal chips were made from silicon, while silicon had poor biocompatibility. UNCD film could be deposited on the silicon chips and a small amount of hydrogen was added to improve the hydrogen doping in the grain boundaries, thus reduced leakage current. This method improved the biocompatibility and survival time of the implantable chips [67]. The UNCD film was applied in the novel approach to treat the retina detachment [68]. In this method, the UNCD was coated on the magnet which attachment on the outside wall of the eye, and then inject the superparamagnetic nanoparticles. Under the external magnet, the magnetic field made the nanoparticles pull the retina back to the wall of the eye. The UNCD coating made the magnet more biocompatible and the attachment could be long-term. Recently, the boron doped porous NCD electrode on retinal implant was mentioned [69]. This retinal prosthesis helped the blind patient, who suffered from hereditary diseases, to restore vision. In addition, the Argonne national laboratory researchers used UNCD film to differentiate embryonic stem cells into retinal photoreceptor cells, which helped those blind people who caused by genetic defects [37]. At present, many researchers had paid attention to the biomedical application of diamond film. For example, Cai et al. reported the application for the NCD film for the cochlear implants [70]. Human inner ear ganglion neurites and the neural progenitor cell could attach well on the NCD surface. Due to titanium and its alloys couldn't endure electrochemical corrosion in the oral environment, Patel et al. investigated the electrochemical behavior of the UNCD film coated on the titanium and its alloy, and found the UNCD coating could be used as the dental implants [71]. In addition, UNCD film can be used as bio sensors enable real-time and continuous detection of molecules for monitoring patient health [72] [73].

\section{Conclusions}

In summary, the CVD nanocrystalline diamond thin film and its biological application were discussed in four parts. The first part covered the characteristics of NCD film and the advantages of MPCVD deposition techniques. The concept, typical morphology, grain size, and the chemical nature of the NCD film were discussed. The NCD film consists of the facet grain, and the chemical structure of the film investigated by Raman spectrum. The D, G band and diamond band were observed in the spectrum. The second part discussed the properties of the NCD film. The properties of the microcrystalline and NCD film were compared in details. The electrical property of the film could be tuned via atomic doping. 
The mechanical property such as hardness and the surface roughness, and the biological property of the film were emphasized. The third part dealt with the microwave plasma chemical vapor deposition, comparing to the hot filament chemical vapor deposition. MPCVD deposited NCD film at low temperature, between $300^{\circ} \mathrm{C}$ and $800^{\circ} \mathrm{C}$, and the relatively high growth rate, highest growth rate for $2.6 \mu \mathrm{m} / \mathrm{h}$ of the film. The final part discussed the biological application. The outstanding mechanical, biocompatibility, bioinert properties made the NCD and UNCD film can be used for medical application, such as, the joint implantation and ophthalmological prosthesis.

This paper discussed the growth of NCD films and its biocompatible application, a research field developed rapidly and have been exploiting extensive applicable prospects. MPCVD is a promising technique in which $\mathrm{n}$ - and p-type NCD films can be formed in deposition process by introducing nitrogen or boron containing precursors. The biocompatible property is related to film structures and the doping types and concentration, thus these studies may provide a foundation for NCD films in applicable biotechnology and bio-devises.

\section{Acknowledgements}

This work was financially supported by the Fundamental Research Funds for the Central Universities (No. XDJK2017D009).

\section{References}

[1] William, G.E. and Kenmore, N.Y. (1962) Synthesis of Diamond. United States Patent Office, Alexandria.

[2] Zarrabian, M., Coulon, N.F., Turban, G. and Marhic, C. (1997) Observation of Nanocrystalline Diamond in Diamondlike Carbon Films Deposited at Room Temperature in Electron Cyclotron Resonance Plasma. Applied Physics Letters, 8, 2535-2537. https://doi.org/10.1063/1.118912

[3] Kouzuma, Y., Teii, K., Uchino, K. and Muraoka, K. (2003) Diamond Nucleation Density as a Function of Ion-Bombardment Energy in Electron Cyclotron Resonance Plasma. Physical Review B, 68, Article ID: 064104.

https://doi.org/10.1103/PhysRevB.68.064104

[4] Williams, O.A., Nesladek, M. and Daenen, M. (2008) Growth, Electronic Properties and Applications of Nanodiamond. Diamond and Related Materials, 17, 1080-1088. https://doi.org/10.1016/j.diamond.2008.01.103

[5] Podgursky, V., Bogtaov, A., Sedov, V. and Sildos, I. (2015) Growth Dynamics of Nanocrystalline Diamond Films Produced by Microwave Plasma Enhanced Chemical Vapor Deposition in Methane/Hydrogen/Air Mixture: Scaling Analysis of Surface Morphology. Diamond and Related Materials, 58, 172-179.

https://doi.org/10.1016/j.diamond.2015.07.002

[6] Kuzmany, H., Pfeiffer, R. and Salk, N. (2004) The Mystery of the $1140 \mathrm{~cm}^{-1}$ Raman Line in Nanocrystalline Diamond Films. Carbon, 42, 911-917. https://doi.org/10.1016/j.carbon.2003.12.045

[7] Rani, R., Kumar, N., Kozakov, A.T., Googlev, K.A. and Sankaran, K.J. (2015) Superlubrication Properties of Ultra-Nanocrystalline Diamond Film Sliding against a Zirconia Ball. RSC Advances, 5, 100663-100673. 
https://doi.org/10.1039/C5RA18832F

[8] Sankaran, K.J., Kurian, J., Chen, H.C. and Dong, C.L. (2012) Origin of a Needle-Like Granular Structure for Ultrananocrystalline Diamond Films Grown in a $\mathrm{N}_{2} / \mathrm{CH}_{4}$ Plasma. Journal of Physics D: Applied Physics, 45, Article ID: 365303. https://doi.org/10.1088/0022-3727/45/36/365303

[9] Voss, A., Stateva, S.R., Reithmaier, J.P., Apostolova, M.D. and Popov, C. (2017) Patterning of the Surface Termination of Ultrananocrystalline Diamond Films for Guided Cell Attachment and Growth. Surface and Coatings Technology, 321, 229-235. https://doi.org/10.1016/j.surfcoat.2017.04.066

[10] Chu, Y.C., Tu, C.H., Liu, C.P., Tzeng, Y.H. and Auciello, O. (2012) Ultrananocrystalline Diamond Nano-Pillars Synthesized by Microwave Plasma Bias-Enhanced Nucleation and Bias-Enhanced Growth in Hydrogen-Diluted Methane. Journal of Applied Physics, 112, Article ID: 124307. https://doi.org/10.1063/1.4769861

[11] Vlasov, I.I., Goovaerts, E., Ralchenko, V.G., Konov, V.I., Khomich, A.V. and Kanzyuba, M.V. (2007) Vibrational Properties of Nitrogen-Doped Ultrananocrystalline Diamond Films Grown by Microwave Plasma CVD. Diamond and Related Materials, 16, 2074-2077. https://doi.org/10.1016/j.diamond.2007.07.007

[12] Williams, O.A. (2011) Nanocrystalline Diamond. Diamond and Related Materials, 20, 621-640. https://doi.org/10.1016/j.diamond.2011.02.015

[13] Xiao, X., Birrell, J., Gerbi, J.E., Auciello, O. and Carlisle, J.A. (2004) Low Temperature Growth of Ultrananocrystalline Diamond. Journal of Applied Physics, 96, Article ID: 2232. https://doi.org/10.1063/1.1769609

[14] Liu, J., Hei, L.F., Chen, G.C., Li, C.M., Song, J.H. and Tang, W.Z. (2014) Influence of Seeding Pre-Treatments on Mechanical Properties of Ultrananocrystalline Diamond Films on Silicon and $\mathrm{Si}_{3} \mathrm{~N}_{4}$ Substrates. Thin Solid Films, 556, 385-389. https://doi.org/10.1016/j.tsf.2014.01.065

[15] Fendrych, F., Taylor, A., Peksa, L., Kratochvilova, I., Vlcek, J. and Rezacova, V. (2010) Growth and Characterization of Nanodiamond Layers Prepared Using the Plasma-Enhanced Linear Antennas Microwave CVD System. Journal of Physics D-Applied Physics, 43, Article ID: 374018. https://doi.org/10.1088/0022-3727/43/37/374018

[16] Bogdanowicz, R., Smietanam, M., Gnyda, M. and Ficek, M. (2013) Nucleation and Growth of CVD Diamond on Fused Silica Optical Fibres with Titanium Dioxide Interlayer. Physica Status Solidi A-Applications and Materials Science, 210, 1991-1997. https://doi.org/10.1002/pssa.201300096

[17] Li, C.P., Dai, W., Qian, L., Li, M.J., Sun, D.Z. and Gao, C.Y. (2014) Effect of Metallic Seed Layers on the Properties of Nanocrystalline Diamond Films. Diamond and Related Materials, 49, 48-54. https://doi.org/10.1016/j.diamond.2014.08.003

[18] Butler, J.E. and Sumant, A.V. (2008) The CVD of Nanodiamond Materials. Chemical Vapor Deposition, 14, 145-160. https://doi.org/10.1002/cvde.200700037

[19] Guo, J., Wen, B. and Melnik, R. (2011) Molecular Dynamics Study on Diamond Nanowires Mechanical Properties: Strain Rate, Temperature and Size Dependent Effects. Diamond and Related Materials, 20, 551-555. https://doi.org/10.1016/j.diamond.2011.02.016

[20] Vojs, M., Varga, M. and Bachenko, O. (2014) Structural and Electrical Characterization of Diamond Films Deposited in Nitrogen/Oxygen Containing Gas Mixture by Linear Antenna Microwave CVD Process. Applied Surface Science, 312, 226-230. https://doi.org/10.1016/j.apsusc.2014.05.176 
[21] Ikeda, T., Teii, K., Casiraghi, C., Robertson, J. and Ferrai, A.C. (2008) Effect of the $s p^{2}$ Carbon Phase on $n$-Type Conduction in Nanodiamond Films. Journal of Applied Physics, 104, Article ID: 073720. https://doi.org/10.1063/1.2990061

[22] Ficek, M., Sobaszek, M., Gnyba, M. and Ryl, J. (2016) Optical and Electrical Properties of Boron Doped Diamond Thin Conductive Films Deposited on Fused Silica Glass Substrates. Applied Surface Science, 387, 846-856. https://doi.org/10.1016/j.apsusc.2016.06.165

[23] Neto, M.A., Pato, G., Bundaleski, N., Teodoro, O.M.N.D. and Fernandes, A.J.S. (2016) Surface Modifications on As-Grown Boron Doped CVD Diamond Films Induced by the $\mathrm{B}_{2} \mathrm{O}_{3}$-Ethanol-Ar System. Diamond and Related Materials, 64, 89-96. https://doi.org/10.1016/j.diamond.2016.02.001

[24] Koinkar, P.M., Patil, S.S., Kim, T.G. and Yonekura, D. (2011) Enhanced Field Emission Characteristics of Boron Doped Diamond Films Grown by Microwave Plasma Assisted Chemical Vapor Deposition. Applied Surface Science, 257, 1854-1858. https://doi.org/10.1016/j.apsusc.2010.08.121

[25] Hu, Q., Joshi, R.K. and Kumar, A. (2010) Electrons Diffusion Study on the Nitrogen-Doped Nanocrystalline Diamond Film Grown by MPECVD Method. Applied Surface Science, 256, 6233-6236. https://doi.org/10.1016/j.apsusc.2010.03.147

[26] Yuan, W., Fang, L.P., Feng, Z., Chen, Z.X., Wen, J.W. and Xiong, Y. (2016) Highly Conductive Nitrogen-Doped Ultrananocrystalline Diamond Films with Enhanced Field Emission Properties: Triethylamine as a New Nitrogen Source. Journal of Materials Chemistry C, 4, 4778-4785. https://doi.org/10.1039/C6TC00087H

[27] Ariano, P., Baldeli, P., Carbone, E., Gilarfdino, A., Giudice, A.L. and Lovisolo, D. (2005) Cellular Adhesion and Neuronal Excitability on Functionalised Diamond Surfaces. Diamond and Related Materials, 14, 669-674. https://doi.org/10.1016/j.diamond.2004.11.021

[28] Müller, R., Adamschik, M., Steidl, D., Kohn, E., Thamasett, S. and Stiller, S. (2004) Application of CVD-Diamond for Catheter Ablation in the Heart. Diamond and Related Materials, 13, 1080-1083. https://doi.org/10.1016/j.diamond.2003.12.012

[29] Yang, L., Sheldon, B.W. and Webster, T.J. (2009) The Impact of Diamond Nanocrystallinity on Osteoblast Functions. Biomaterials, 30, 3458-3465. https://doi.org/10.1016/j.biomaterials.2009.03.014

[30] Broz, A., Ukraintsev, E., Kromka, A., Rezek, B. and Kalbacova, M.H. (2017) Osteoblast Adhesion, Migration, and Proliferation Variations on Chemically Patterned Nanocrystalline Diamond Films Evaluated by Live-Cell Imaging. Journal of Biomedical Materials Research Part A, 105, 1469-1478. https://doi.org/10.1002/jbm.a.35969

[31] Smirnov, W., Kriele, A., Yang, N. and Nebel, C.E. (2010) Aligned Diamond Nano-Wires: Fabrication and Characterisation for Advanced Applications in Bio- and Electrochemistry. Diamond and Related Materials, 19, 186-189. https://doi.org/10.1016/j.diamond.2009.09.001

[32] Nebel, C.E., Yang, N., Uetsuka, H., Osawa, H. and Tokuda, N. (2009) Diamond Nano-Wires, a New Approach towards Next Generation Electrochemical Gene Sensor Platforms. Diamond and Related Materials, 18, 910-917. https://doi.org/10.1016/j.diamond.2008.11.024

[33] Verdanova, M., Rezek, B., Broz, A. and Ukraintsev, E. (2016) Nanocarbon Allotropes-Graphene and Nanocrystalline Diamond-Promote Cell Proliferation. Smal, 12, 2499-2509. 
[34] Alcaide, M., Papaioannou, S., Taylor, A. and Fekete, L. (2016) Resistance to Protein Adsorption and Adhesion of Fibroblasts on Nanocrystalline Diamond Films: The Role of Topography and Boron Doping. Journal of Materials Science: Materials in Medicine, 27, 90. https://doi.org/10.1007/s10856-016-5696-3

[35] Fromell, K., Karlsson, P., Larsson, M., Nikolajeff, K. and Balter, L. (2012) Designed Protein Binders in Combination with Nanocrystalline Diamond for Use in High-Sensitivity Biosensors. Analytical and Bioanalytical Chemistry, 404, 1643-1651. https://doi.org/10.1007/s00216-012-6245-7

[36] Ariano, P., Budnyk, O., Dalmazzo, S. and Lovisolo, D. (2009) On Diamond Surface Properties and Interactions with Neurons. The European Physical Journal E, 30, 149-156. https://doi.org/10.1140/epje/i2009-10520-9

[37] Park, K.H. and Na, K. (2008) Effect of Growth Factors on Chondrogenic Differentiation of Rabbit Mesenchymal Cells Embedded in Injectable Hydrogels. Journal of Bioscience and Bioengineering, 106, 74-79.

[38] Bajaj, P., Akin, D. and Gupta, A. (2007) Ultrananocrystalline Diamond Film as an Optimal Cell Interface for Biomedical Applications. Biomedical Microdevices, 9, 787-794. https://doi.org/10.1007/s10544-007-9090-2

[39] Specht, C.G., Williams, O.A., Jackman, R.B. and Schoepfer, R. (2004) Ordered Growth of Neurons on Diamond. Biomaterials, 25, 4073-4078.

https://doi.org/10.1016/j.biomaterials.2003.11.006

[40] Shi, B., Jin, Q.L., Chen, L.H. and Auciello, O. (2009) Fundamentals of Ultrananocrystalline Diamond (UNCD) Thin Films as Biomaterials for Developmental Biology: Embryonic Fibroblasts Growth on the Surface of (UNCD) Films. Diamond and Related Materials, 18, 596-600. https://doi.org/10.1016/j.diamond.2008.09.016

[41] Shi, B., Jin, Q.L., Chen, L.H., Woods, A.S., Schultz, A.J. and Auciello, O. (2012) Cell Growth on Different Types of Ultrananocrystalline Diamond Thin Films. Journal of Functional Biomaterials, 3, 588-600. https://doi.org/10.3390/jfb3030588

[42] Chen, Y.C., Lee, D.C. and Hsiao, C.Y. (2009) The Effect of Ultra-Nanocrystalline Diamond Films on the Proliferation and Differentiation of Neural Stem Cells. Biomaterials, 30, 3428-3435. https://doi.org/10.1016/j.biomaterials.2009.03.058

[43] Tong, W., Fox, K., Zamani, A., Turnley, A.M., Ganesan, K. and Ahood, A. (2016) Optimizing Growth and Post Treatment of Diamond for High Capacitance Neural Interfaces. Biomaterials, 104, 32-42. https://doi.org/10.1016/j.biomaterials.2016.07.006

[44] Vaitkuviene, A., McDonald, M., Vahidpour, F., Noben, J.P., Sanen, K. and Ameloot, M. (2015) Impact of Differently Modified Nanocrystalline Diamond on the Growth of Neuroblastoma Cells. New Biotechnology, 32, 7-12. https://doi.org/10.1016/j.nbt.2014.06.008

[45] Lechleitner, T., Klauser, F., Seppi, T., Lechner, J., Jennings, P. and Percp, P. (2008) The Surface Properties of Nanocrystalline Diamond and Nanoparticulate Diamond Powder and Their Suitability as Cell Growth Support Surfaces. Biomaterials, 29, 4275-4284. https://doi.org/10.1016/j.biomaterials.2008.07.023

[46] Amaral, M., Gomes, P.S., Lopes, M.A., Santors, J.D. and Silva, R.F. (2009) Cytotoxicity Evaluation of Nanocrystalline Diamond Coatings by Fibroblast Cell Cultures. Acta Biomaterialia, 5, 755-763. https://doi.org/10.1016/j.actbio.2008.08.015

[47] Auciello, O. and Sumant, A.V. (2010) Status Review of the Science and Technology of Ultrananocrystalline Diamond (UNCD ${ }^{\mathrm{TM}}$ ) Films and Application to Multifunctional Devices. Diamond and Related Materials, 19, 699-718. 
https://doi.org/10.1016/j.diamond.2010.03.015

[48] Subramanian, K., Kang, W.P., Davidson, J.L. and Hofmeister, W.H. (2005) The Effect of Growth Rate Control on the Morphology of Nanocrystalline Diamond. Diamond and Related Materials, 14, 404-410. https://doi.org/10.1016/j.diamond.2005.01.027

[49] Das, D. and Banerjee, A. (2015) Further Improvements of Nano-Diamond Structures on Unheated Substrates by Optimization of Parameters with Secondary Plasma in MW-PECVD. Surface and Coatings Technology, 272, 357-365.

https://doi.org/10.1016/j.surfcoat.2015.03.042

[50] Sankaran, K.J., Huang, B.R., Saravanan, A., Manoharan, D. and Tai, N.H. (2016) Nitrogen Incorporated Ultrananocrystalline Diamond Microstructures from Bias-Enhanced Microwave $\mathrm{N}_{2} / \mathrm{CH}_{4}$-Plasma Chemical Vapor Deposition. Plasma Processes and Polymers, 13, 419-428. https://doi.org/10.1002/ppap.201500079

[51] Lin, I.N., Chen, H.C., Wang, C.S., Lee, Y.R. and Lee, C.Y. (2011) Nanocrystalline Diamond Microstructures from $\mathrm{Ar} / \mathrm{H}_{2} / \mathrm{CH}_{4}$-Plasma Chemical Vapour Deposition. CrystEngComm, 13, 6082-6089. https://doi.org/10.1039/c1ce05517h

[52] Tang, C.J., Pereira, S.M.S., Feranadaes, A.J.S., Neves, A.J., Gracio, J. and Bdikin, I.K. (2009) Synthesis and Structural Characterization of Highly $<100>$-Oriented $\{100\}$-Faceted Nanocrystalline Diamond Films by Microwave Plasma Chemical Vapor Deposition. Journal of Crystal Growth, 311, 2258-2264.

https://doi.org/10.1016/j.jcrysgro.2009.01.130

[53] Tang, C.J., Fernandes, A.J.S., Granada, M., Leitao, J.P., Pereira, S. and Jiang, X.F. (2015) High Rate Growth of Nanocrystalline Diamond Films Using High Microwave Power and Pure Nitrogen/Methane/Hydrogen Plasma. Vacuum, 122, 342-346. https://doi.org/10.1016/j.vacuum.2015.03.021

[54] Harada, Y., Hishinuma, R., Terashima, C., Uetsuka, H., Nakata, K. and Kondo, T. (2016) Rapid Growth of Diamond and Its Morphology by In-Liquid Plasma CVD. Diamond and Related Materials, 63, 12-16. https://doi.org/10.1016/j.diamond.2015.10.009

[55] Tang, C.J., Fernandes, A.J.S., Jiang, X.F., Pinto, J.L. and Ye, H. (2016) Impact of High Microwave Power on Hydrogen Impurity Trapping in Nanocrystalline Diamond Films Grown with Simultaneous Nitrogen and Oxygen Addition into Methane/Hydrogen Plasma. Journal of Crystal Growth, 434, 36-41.

https://doi.org/10.1016/j.jcrysgro.2015.10.037

[56] Liu, C., Wang, J.H. and Weng, J. (2015) Growth of Micro- and Nanocrystalline Dual Layer Composite Diamond Films by Microwave Plasma CVD: Influence of $\mathrm{CO}_{2}$ Concentration on Growth of Nano-Layer. Journal of Crystal Growth, 410, 30-34. https://doi.org/10.1016/j.jcrysgro.2014.10.040

[57] Tien, H.W., Lee, C.Y., Lin, I.I. and Chen, Y.C. (2017) Long Term in Vivo Functional Stability and Encapsulation Reliability of Using Ultra-Nanocrystalline Diamond as an Insulating Coating Layer for Implantable Microchips. Journal of Materials Chemistry B, 5, 3706-3717. https://doi.org/10.1039/C7TB00867H

[58] Salgueiredo, E., Almeida, F.A., Amaral, M., Fernandes, A.J.S. and Costa, F.M. (2009) CVD Micro/Nanocrystalline Diamond (MCD/NCD) Bilayer Coated Odontological Drill Bits. Diamond and Related Materials, 18, 264-270. https://doi.org/10.1016/j.diamond.2008.08.014

[59] Skoog, S.A., Lu, Q.J., Malinauskas, R.A. and Sumant, A.V. (2017) Effects of Nanotopography on the in Vitro Hemocompatibility of Nanocrystalline Diamond Coat- 
ings. Journal of Biomedical Materials Research Part A, 105, 253-264. https://doi.org/10.1002/jbm.a.35872

[60] Xiao, X.C., Wang, J., Liu, C., Carlisle, J.A., Mech, B., Greenberg, R., Guven, D. and Freda, R. (2006) In Vitro and in Vivo Evaluation of Ultrananocrystalline Diamond for Coating of Implantable Retinal Microchips. Journal of Biomedical Materials Research Part B: Applied Biomaterials, 77B, 273-281.

https://doi.org/10.1002/jbm.b.30448

[61] Hajra, M., Hunt, C.E., Ding, M., Auciello, O., Carlisle, J. and Gruen, D.M. (2003) Effect of Gases on the Field Emission Properties of Ultrananocrystalline Diamond-Coated Silicon Field Emitter Arrays. Journal of Applied Physics, 94, 4079-4083. https://doi.org/10.1063/1.1594268

[62] Wang, C.F., Choi, Y.S., Lee, J.C. and Hu, E.L. (2007) Observation of Whispering Gallery Modes in Nanocrystalline Diamond Microdisks. Applied Physics Letters, 90, Article ID: 081110. https://doi.org/10.1063/1.2709626

[63] Wang, C.F., Hanson, R., Awschalom, D.D., Hu, E.L. and Feygelson, T. (2007) Fabrication and Characterization of Two-Dimensional Photonic Crystal Microcavities in Nanocrystalline Diamond. Applied Physics Letters, 91, Article ID: 201112. https://doi.org/10.1063/1.2813023

[64] Tadjer, M.J., Hobart, K.D., Caldwell, J.D. and Butler, J.E. (2007) Nanocrystalline Diamond Films as UV-Semitransparent Schottky Contacts to $4 H$-SiC. Applied Physics Letters, 91, Article ID: 163508. https://doi.org/10.1063/1.2800886

[65] Amaral, M., Abreu, C.S. and Oliveira, F.J. (2008) Tribological Characterization of NCD in Physiological Fluids. Diamond and Related Materials, 17, 848-852. https://doi.org/10.1016/j.diamond.2007.12.070

[66] Jozwik, K. and Karczemska, A. (2007) The New Generation Ti6AI4V Artificial Heart Valve with Nanocrystalline Diamond Coating on the Ring and with Derlin Disc after Long-Term Mechanical Fatigue Examination. Diamond and Related Materials, 16, 1004-1009. https://doi.org/10.1016/j.diamond.2006.12.051

[67] Vargas, J.M. and Zysler, R.D. (2005) Tailoring the Size in Colloidal Iron Oxide Magnetic Nanoparticles. Nanotechnology, 16, 1474-1476.

https://doi.org/10.1088/0957-4484/16/9/009

[68] Hadjinicolaou, A.E., Leung, R.T., Garrett, D.J., Ganesan, K. and Fox, K. (2012) Electrical Stimulation of Retinal Ganglion Cells with Diamond and the Development of an All Diamond Retinal Prosthesis. Biomaterials, 33, 5812-5820. https://doi.org/10.1016/j.biomaterials.2012.04.063

[69] Hebert, C., Cottance, M., Degardin, J., Scorsone, E. and Rousseau, L. (2016) Monitoring the Evolution of Boron Doped Porous Diamond Electrode on Flexible Retinal Implant by OCT and in Vivo Impedance Spectroscopy. Materials Science \& Engineering: $C, 69,77-84$.

https://doi.org/10.1016/j.msec.2016.06.032

[70] Cai, Y.X., Edin, F., Jin, Z., Alexsson, A., Gudjonsson, O. and Liu, W. (2016) Strategy Towards Independent Electrical Stimulation from Cochlear Implants: Guided Auditory Neuron Growth on Topographically Modified Nanocrystalline Diamond. Acta Biomaterialia, 31, 211-220. https://doi.org/10.1016/j.actbio.2015.11.021

[71] Patel, B., Martinez, A.C.D., Gurman, P., Auciello, O. and Barao, V. (2017) Ultrananocrystalline Diamond Coatings for the Dental Implant: Electrochemical Nature. Surface Innovations, 5, 106-117. https://doi.org/10.1680/jsuin.16.00023

[72] Skoog, S.A., Miller, P.R., Boehm, R.D., Sumant, A.V. and Polsky, R. (2015) Nitro- 
gen-Incorporated Ultrananocrystalline Diamond Microneedle Arrays for Electrochemical Biosensing. Diamond and Related Materials, 54, 39-46.

https://doi.org/10.1016/j.diamond.2014.11.016

[73] Siddiqui, S., Dai, Z., Stavis, C.J., Zeng, H.J., Moldovan, N. Hamers, R.J. and Carlsile, J.A. (2012) A Quantitative Study of Detection Mechanism of a Label-Free Impedance Biosensor Using Ultrananocrystalline Diamond Microelectrode Array. Biosensors and Bioelectronics, 35, 284-290. https://doi.org/10.1016/j.bios.2012.03.001 